\title{
DISEÑO DE UN SISTEMA ALIMENTADOR PARA UN HORNO ROTATORIO EN LA PRODUCCIÓN DE FOSFATO EN NORTE DE SANTANDER
}

\section{DESIGN OF A FEEDING SYSTEM FOR A ROTARY OVEN IN THE PRODUCTION OF PHOSPHATE IN NORTE OF SANTANDER}

\author{
*MSc. Eder Flórez Solano, *MSc (c). Ricardo Andrés García León, \\ *MSc Edgar Antonio Sánchez Ortiz \\ *Universidad Francisco de Paula Santander Ocaña. \\ Facultad de Ingenierías, Grupo de Investigación INGAP. \\ Vía Acolsure, Sede el Algodonal - Ocaña, Norte de Santander, Colombia. \\ Tel.: +57 5690088 Ext. 191. \\ E-mail: (enflorez, ragarcial, easanchezo) @ufpso.edu.co.
}

\begin{abstract}
Resumen: El empleo de fertilizantes artificiales en forma de minerales de ácido fosfórico, sales de fosfato y sus derivados ha aumentado en la última década, debido al consumo por parte de los fabricantes y clientes. Durante las décadas recientes las distintas fábricas de fosfatos han hecho grandes avances para recortar los costos, tanto de producción como de distribución, lo que ha permitido que los ácidos fosfóricos así como de sus sales, se empleen en amplios campos, logrando también la introducción de nuevos derivados. Es de esta manera que se requieren desarrollos de industrias eficientes del fosforo, tanto respecto a sus antiguos compuestos como a los nuevos. Actualmente las empresas dedicadas a la fabricación de fertilizantes en la región norte santandereana, son unas de las más importantes encargadas de la explotación, procesamiento y comercialización del mineral de fosforo, por esta razón se hace necesaria la proyección del desarrollo de su planta física, la tecnificación de sus equipos y el mejoramiento de sus procesos con la finalidad de aumentar la producción y por consiguiente el crecimiento económico, contribuyendo de esta manera al abastecimiento en la región y en otras zonas del país. Por lo anterior, se planteó el diseño de un sistema alimentador en donde se analizaron los elementos mecánicos que hacen parte del proceso de calentamiento del fosfato, con la finalidad de tecnificar la etapa de alimentación y de esta manera disminuir costos y por consiguiente aumentar la producción y calidad del producto.
\end{abstract}

Palabras clave: Sistema Alimentador, Horno, Fosforo, Fertilizante, Mineral, Diseño.

Abstract: The use of artificial fertilizers in the form of phosphoric acid minerals, phosphate salts and their derivatives has increased over the last decade due to consumption by manufacturers and customers. During recent decades the different phosphate factories have made great strides in cutting costs, both production and distribution, which has allowed phosphoric acids as well as their salts to be used in wide fields. New derivatives. It is in this way that efficient phosphorus industry developments are required, both with regard to their old compounds as well as to the new ones. At the moment, the companies dedicated to the manufacture of fertilizers in the northern region of Santander, are one of the most important in charge of the exploitation, processing and commercialization of phosphorus ore, for this reason it is necessary the projection of the development of its physical plant, the Upgrading its equipment and improving its processes in order to increase production and consequently economic growth, thus contributing to supply in the region and other areas of the country. Due to the above, the design of a feeder system was analyzed in which the mechanical elements that are part of the phosphate heating process were analyzed, in order to technify the feeding stage and in this way to reduce costs and consequently to increase production and product quality.

Keywords: System Feeder, Furnace, Phosphorus, Fertilizer, Mineral, Design. 


\section{INTRODUCCIÓN}

En cuanto a su trascendencia a nivel industrial cabe destacar su presencia en diferentes procesos tecnológicos: la manipulación, transporte, almacenamiento, acopio, mezcla y empaquetamiento de polvos, piedras, combustibles sólidos, harinas, sales, granos y semillas son prácticas comunes en las industrias farmacéutica, cosmética, de construcción y agroalimenticia entre otras. El tratamiento de los materiales granulares moviliza el $10 \%$ de los recursos energéticos del planeta. Entre los problemas más comunes se encuentran:

- La formación de arcos que conducen a la disminución o bloqueo de flujo a través de los orificios de salida de recipientes, silos y tolvas.

- La tendencia que presentan estos materiales a segregarse debido a la presencia de diferencias en las propiedades y características físicas (forma, tamaño y densidad) de sus constituyentes (los granos). Esta segregación, producto de la manipulación y transporte que someten a los granos a esfuerzo de corte o vibraciones, conspiran contra la obtención de mezclas homogéneas, factor importante en diversas aplicaciones tecnológicas.

- El estudio de estos materiales pretende comprender estas propiedades a fin de elaborar modelos que permitan la descripción de su comportamiento buscando los puntos comunes de los diversos casos particulares.

Es necesaria una mayor comprensión de la física de los materiales granulares a fin de diseñar procesos eficientes en la manipulación de estos sistemas. La siguiente estadística llevada a cabo principalmente en América del Norte ayudará a comprender la importancia:

En la industria química aproximadamente la mitad de los productos y al menos tres cuartos de la materia prima se presenta en forma granular (Nedderman, 2005). Se estima que se invierten 61 billones de dólares en tecnología de partículas (Jaeger, Nagel, Behringer, Hugo, \& Miserables, 1996). Aproximadamente el 1,3\% de la producción de electricidad de los Estados Unidos se emplea en la trituración de partículas y minerales (Budynas \& Nisbett, 2008).

Cada año, solamente en Estados Unidos, fallan más de mil silos, recipientes y tolvas (Knowlton, Klinzing, \& Yang, 1994). El 80\% de las plantas de procesamientos de partículas sólidas en los Estados
Unidos y Canadá presentaron problemas en la manipulación de las mismas. Estos problemas operacionales disminuyeron la eficiencia de diseño entre un $40 \%$ y $50 \%$ (Budynas \& Nisbett, 2008). Resulta evidente que aún una pequeña mejora en la eficiencia en la manipulación de estos materiales tendrá un impacto económico significativo.

\subsection{Consideraciones generales en el transporte de materiales a granel}

Para realizar la comparación de los sistemas de transporte de materiales a granel es importante hacerlo desde el punto de vista de los principales factores que pueden afectar el funcionamiento y la operación de los mismos, entre los cuales tenemos:

La capacidad requerida

La longitud de transporte

La elevación

Las características del material a transportar

Los requerimientos de los procesos

El costo inicial

Motores de transportadores

Equipos auxiliares

Otro problema en el caso de la mayoría de los transportadores es el del corte de la fuerza propulsora, cuando se atasca el transportador. Con frecuencia se usan dispositivos de limitación del esfuerzo de torsión, al igual que controles eléctricos que cortan la alimentación de corriente al motor.

Tabla 1. Alimentadores para materiales a granel.

\section{Características del material}

Materiales finos, de libre fluidez

Materiales no abrasivos y granulares, materiales con algunos trozos.

Materiales difíciles de manejar porque son muy calientes, abrasivos, aterronados o fibrosos.

Materiales pesados, con terrones $\mathrm{o}$ abrasivos, similares a

\section{Tipo de alimentador}

De barras, de banda,

oscilador o vibrador, de paletas giratorias, de tornillo.

De banda articulada, de barras, de banda, oscilador o vibrador, de movimiento reciprocante, de placa giratoria, de tornillo.

De banda articulada, de barras, de banda, oscilador o vibrador, de movimiento reciprocante. De banda articulada, oscilador o vibrador, de movimiento 
minerales o guijarros. reciprocante.

Fuente: ("Link-Belt Cranes," 2015).

\section{MATERIALES Y MÉTODOS}

La empresa cuenta con un cargador que no realiza su función completamente debido al mal diseño de la tolva de recepción, la cual no cuenta con un acceso fácil para la máquina, obligando a un operario a efectuar una labor ardua, alimentar nuevamente la tolva mediante la utilización de una pala, ya que el cargador solo acerca el material a las proximidades de la tolva, dejando el resto del trabajo al operario, quien podría con el tiempo desarrollar una enfermedad profesional que demandaría posteriores gastos de seguridad social a la empresa. Por eso se tendrán en cuenta las especificaciones de la pala del cargador para diseñar una tolva cuyo borde superior esté a nivel con el nivel 3 o área de descarga, provista de cajón antiderrame y con las tolerancias apropiadas en cuanto a la altura y ancho de la pala para que el cargador realice la descarga directa del material sobre la tolva, además de una estructura que garantice la seguridad del sistema y la inclusión de un promotor de flujo (sistema vibrante) con el fin de evitar los problemas de flujo (García-León, Flórez Solano, \& Acosta Pérez, 2015).

Con el fin de evitar los problemas que presenta el alimentador actual de la planta se recurrió a las recomendaciones dadas por la empresa Jenike \& Johanson (Marinelli \& Carson, 1992), donde se da a conocer una solución muy eficaz para obtener una extracción más confiable de la descarga de la tolva.

El alimentador consta de tres secciones, las cuales varían su paso progresivamente sobre la extracción hasta la descarga:

Sección A: ubicada bajo la salid de la tolva, se encarga de realizar la extracción del material de forma directa mediante la implementación de la mitad del paso de la sección de extensión del transportador, esto con el fin de evitar zonas muertas $y$ obtener un mejor arrastre $o$ desplazamiento.

Sección B: es una zona de transición donde el paso aumenta a 2/3 del paso estándar de la sección del transportador.

Sección C: es la extensión desde la zona de transición hasta el punto de descarga, que se caracteriza por cumplir la función de dosificador y por tener un paso estándar ( paso = diámetro).

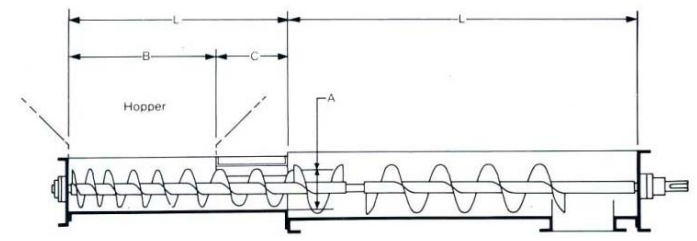

Figura 1. Vista general de las secciones del alimentador.

Fuente: Elaboración propia.

La artesa es la sección en forma de canal (U) que se encarga de contener el tornillo y facilitar el desplazamiento del material, para una mayor comodidad y para efectos de poder realizar limpieza y mantenimiento, se seleccionan perchas en los extremos de la artesa que puedan atornillarse y así lograr el despiece para esta serie de operaciones (Torres et al., 2013).

La forma de las aspas o hélices del tornillo son de forma estándar y sin ninguna modificación, debido a que sólo se requiere desplazar materia prima y no exige ninguna otra condición para el proceso.

\section{Tornillo de mano derecha.}

En la sección A y B, el nivel de llenado es de $95 \%$ y en la sección $\mathrm{C}$ se siguen las recomendaciones para el tipo de material manejado.

A continuación se presenta el desarrollo del procedimiento de diseño del alimentador sinfín propuesto por Martín Sprockets and Grears Co (Martin, 2015), el cual se rige por los parámetros de la norma CEMA.

\subsection{Establecimiento de los factores conocidos.}

Tipo de material a ser manejado. Según el laboratorio de química de Fosfonorte S.A., el nombre del material es Roca Fosfórica, designada como Fluoropatita, cuya fórmula química es Caro $\mathrm{F}_{2}\left(\mathrm{P}_{2} \mathrm{O}_{5}\right)_{6}$.

Máximo tamaño de los trozos duros. Según los resultados obtenidos del estudio de granulometría realizadas a una muestra de material tipo agronómico de maza $\mathrm{m}=105.3$ gramos en el laboratorio de suelos civiles de la universidad Francisco de Paula Santander (ver anexo J), Se observa que el tamaño máximo de los trozos más grandes es del tamaño del tamiz \#8. 
Porcentaje de trozos duros por volumen. En el tamiz \#8, el peso retenido es $0.10 \mathrm{~g}$. El porcentaje referido acumulado es 0.09 , el $99.91 \%$ del material pasa.

La capacidad requerida. De acuerdo a la producción de la planta, para el material tipo agronómico en medio turno (4 horas) el total es de 12 toneladas. Buscando un óptimo rendimiento del proceso de alimentación y asegurando la descarga efectiva de la tolva mediante la implementación de la tolva, se toma como capacidad requerida:

$\mathrm{C}=12$ ton $/ \mathrm{h}=13215,85 \mathrm{Lb} / \mathrm{h}$

Flujo volumétrico. Teniendo la capacidad (lb/h) y la densidad del material, $1830 \mathrm{~kg} / \mathrm{m} 3=113,51$ Lb/píe 3, se obtiene el flujo volumétrico.

$\mathrm{V}=\mathrm{m} / \rho=13215,85 \mathrm{Lb} / \mathrm{h}=116.42$ píe $3 / \mathrm{h}$

Distancia a la que el material se conduce. Conocida la distancia desde la boca de entrada del horno al extremo de carga de la tolva, se resta la longitud del extremo derecho de la boca de salida de la tolva al muro y se obtiene:

$\mathrm{L}_{\text {Total }}=5.35 \mathrm{~m}-1.125 \mathrm{~m}=4.225 \mathrm{~m} \cong 13.53$ píe

Otros factores adicionales que pueden afectar la operación del alimentador. La humedad relativa del sector puede afectar la humedad propia del material, lo que puede acarrear un serio problema de abrasión que debe tenerse en cuenta en los siguientes pasos del diseño.

\subsection{Clasificación del material.}

Para clasificar el material tipo industrial se tiene en cuenta las condiciones presentadas en la carta de códigos de clasificación de materiales, de lo que resulto la siguiente información:

\section{Densidad a granel: $113.51 \mathrm{Lb} /$ pié $^{3}$}

Tamaño: como el $99.91 \%$ del material se clasificó como fino en el estudio granulométrico, debido a que éste porcentaje tiene un tamaño de grano promedio de $2 \mathrm{~mm}=0.078$ pulg, de la tabla se comprueba.

Fino $\rightarrow$ Tamiz \#6 (0.132 pug) o menos $=86$

Fluidez: se considera que la fluidez del material es promedio $=3$
Abrasividad: debido a las condiciones de humedad y humedad relativa mencionadas, se considera extremadamente abrasivo $=7$.

Propiedades misceláneas o de riego: el material no presenta propiedades peligrosas, por tanto no se consideran.

Así el código del material según la anterior tabla es: B6-3-7

Este código se comprueba por medio de la tabla presentada en el anexo $\mathrm{K}$ donde por medio de la densidad y la caracterización como arena del estudio granulométrico, se toma:

Arena fosfórica (Phosphate Sand), con una densidad de 90 - $100 \mathrm{Lb} / \mathrm{pie}^{3}$, cuyo código intermedio de material es B6-37. Por tanto se concluye que el material está bien clasificado.

De la tabla se obtiene además:

Código de selección de cojinetes= serie $\mathrm{H}$

Código de selección de componentes $=$ serie 3

Factor de material $=\mathrm{Fm}=2$

Carga de la artesa en la sección de transporte $=$ $15 \%$

\subsection{Determinación de la capacidad de diseño.}

La capacidad equivalente está fundamentada en la capacidad requerida multiplicada por los factores de capacidad, por medio de la siguiente expresión:

(Capacidad equivalente. $($ Píe $/ \mathrm{h}))=($ Capacidad requerida (píe $3 / \mathrm{h})$. (( $\left.\left.\mathrm{C}_{\mathrm{f} 1}\right) \cdot\left(\mathrm{C}_{\mathrm{F} 2}\right) .\left(\mathrm{C}_{\mathrm{F} 3}\right)\right)$

Donde:

$\mathrm{C}_{\mathrm{F} 1}=$ factor de paso del tornillo

$\mathrm{C}_{\mathrm{F} 2}=$ factor de la forma de la hélice

$\mathrm{C}_{\mathrm{F} 3}=$ factor del número de hélices

Como la sección del alimentador se diseña con paso medio (1/2 diámetro) se toma la opción crítica. Luego $\mathrm{C}_{\mathrm{F} 1}=2$.

Como no se utiliza ninguna de las modificaciones planteadas, $\mathrm{C}_{\mathrm{F} 2}=1$.

En la sección del alimentador, se tiene paso medio por tanto, habrá dos hélices por paso, luego $\mathrm{C}_{\mathrm{F} 3}=$ 1.16 .

Entonces la capacidad de diseño es:

$\mathrm{C}_{\text {equiv }}=\left(116.42 \mathrm{pí}^{3} / \mathrm{h}\right) * 2 * 1 * 1.16$

$\mathrm{C}_{\text {equiv }}=270.09 \mathrm{pí}^{3} / \mathrm{h}$ 


\subsection{Determinación del diámetro y la velocidad}

Con la capacidad equivalente determinada, se obtiene la capacidad estándar a la máxima velocidad, el diámetro y las dimensiones recomendadas para las secciones del alimentador y la extensión del transportador:

Capacidad $=336 \mathrm{pí}^{3} / \mathrm{h}$

Velocidad máxima $=70 \mathrm{rpm}$

$\mathrm{C}^{\prime}=4.8 \mathrm{pí}^{3} / \mathrm{h}$ en $1 \mathrm{rpm}$

Diámetro recomendado $=6$ pulgadas

El tamaño de grano del material no debe exceder a $3 / 4 "=19 \mathrm{~mm}$. El material cumple con todas las condiciones, por lo que se tomaron las siguientes consideraciones:

$\mathrm{B}=36 \mathrm{pul}=91.44 \mathrm{~cm}$ (boca de entrada del material). Esta es la sección donde el paso recomendado para el alimentador es $\rho=1 / 2, d=3$ pul.

$\mathrm{C}=12 \mathrm{pul}=30.48 \mathrm{~cm}$ (zona del incremento del paso). En este sección se recomienda el uso de un incremento en el paso, $\rho=2 / 3 \mathrm{~d}=4$ pul.

$\mathrm{D}=7$ pul. Distancia del centro del eje a la parte superior de la artesa.

$\mathrm{E}=14$ pul. Ancho de la artesa en la parte superior.

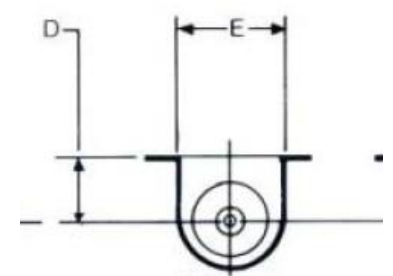

Figura 2. Sección transversal de la artesa.

Fuente: Elaboración propia.

En la sección de transporte, para 15\% recomendado para el material el nuevo porcentaje de carga es $12 \%$, con un paso estándar $\mathrm{p}=\mathrm{d}$.

La longitud de la extensión del transportador está dada por:

$\mathrm{L}=\mathrm{L}_{\text {total }}-\mathrm{L}_{\text {alimentador }}$

$\mathrm{L}=\mathrm{L}_{\text {total }}-(\mathrm{B}+\mathrm{C})=13.53$ píe $-(3+1)$ píe $=9.53$

píe

Verificación del tamaño mínimo debido a las limitaciones del tamaño de grano del material.
Primero se analiza el material según la siguiente clasificación:

Clase 1: mezcla de trozos duros y finos en que no más del $10 \%$ son trozos que van del tamaño máximo a la mitad del tamaño máximo y $90 \%$ son los trozos más pequeños que la mitad del máximo.

Clase 2: mezcla de trozos duros y finos en que no más del $25 \%$ van del tamaño máximo a la mitad del máximo y $75 \%$ son trozos más pequeños que la mitad del máximo.

Clase 3: una mezcla de trozos en que el 95\% o más son trozos que van del tamaño del máximo a la mitad del máximo y 5\% o menos son trozos menores del décimo del tamaño máximo.

Del estudio granulométrico se observa que más del 95\% del material tienen un tamaño que va de 0.4 a $2 \mathrm{~mm}$, y el resto presenta tamaños que no superan el tamiz \#8.

Por esto se clasifica como clase III.

De la tabla M1 (del anexo M) se toman las dimensiones recomendadas para el eje del alimentador, el claro y la holgura radial.

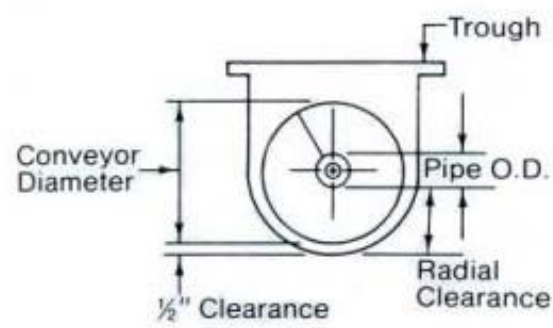

Figura 3. Holgura y claro radial entre el aspa y la artesa.

Fuente: Elaboración propia.

Holgura radial $=1 / 2$ pulg.

Claro radial $=25 / 16$ pulg.

\section{RESULTADOS Y DISCUSIÓN}

\subsection{Cálculo de la potencia requerida}

La potencia requerida para operar un tornillo alimentador está basada en la apropiada, uniforme y regular tasa de alimentación y otros criterios de diseño que se enuncian a continuación:

La potencia debe ser la suma total de las potencias para vencer la inercia tanto del alimentador como de la extensión del transportador y la potencia para vencer la fricción del material cargado en las dos secciones mencionadas. 
Potencia para vencer la inercia del alimentador al vacío:

$$
\mathrm{Hp}_{\mathrm{a}}=\underline{\mathrm{L}}_{1} \frac{* \mathrm{~N} * \mathrm{~F}_{\mathrm{d}}}{1.000} \frac{\mathrm{F}_{\mathrm{b}}}{200} \frac{\mathrm{F}_{\mathrm{f}}}{}
$$

Donde:

$\mathrm{L}_{1}=$ longitud de la sección del alimentador, píe.

$\mathrm{L}_{1}=\mathrm{B}+\mathrm{C}=4$ píe

$\mathrm{F}_{\mathrm{f}}=$ factor de aspa, de la tabla N1 (anexo N), para aspa estándar y $95 \%$ carga $\mathrm{F}_{\mathrm{f}}=1$

$\mathrm{F}_{\mathrm{d}}=$ factor de diámetro del tornillo, de la tabla N2

(anexo N) para $\varnothing=6 " \rightarrow F_{d}=18$

$\mathrm{N}=$ máxima velocidad, $\mathrm{rpm}$

Reemplazando se obtiene:

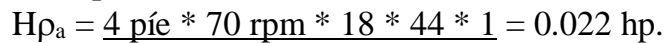

$$
1.000 .000
$$

\section{Potencia para vencer la fricción del material en} el alimentador:

$\mathrm{Hp}_{\mathrm{b}}=\frac{\mathrm{C} * \mathrm{~W} * \mathrm{~L} * \mathrm{~F}_{\mathrm{m}}}{1.000 .000}$

Donde:

$\mathrm{C}=$ capacidad requerida, $\mathrm{pí}^{3} / \mathrm{h}$

$\mathrm{W}=$ densidad a granel del material, Lb/píe

$\mathrm{F}_{\mathrm{m}}=$ factor del material

$\mathrm{L}_{\mathrm{f}}=$ longitud corregida de la sección, píe

Por recomendación $=\mathrm{L}_{\mathrm{f}}=\mathrm{L}_{1}+\mathrm{B} / 6+\mathrm{C} / 12$

Luego $\mathrm{L}_{\mathrm{f}}=4+3 / 6+1 / 12=4.58$ píe

$\mathrm{Hp}_{\mathrm{b}}=\underline{336 \mathrm{pí}^{3} / \mathrm{h} * 113.51 \mathrm{Lb} / \mathrm{pí}^{3} * 4.58 \text { píe } * 2}$ 1.000 .000

$\mathrm{Hp}_{\mathrm{b}}=0.349 \mathrm{hp}$.

Potencia para vencer la inercia de la extensión del transportador al vacío:

$\mathrm{Hp}_{\mathrm{c}}=\frac{\mathrm{L} * \mathrm{~N} * \mathrm{~F}_{\mathrm{d}} * \mathrm{~F}_{\mathrm{b}} * \mathrm{~F}_{\mathrm{f}}}{1.000 .000}$

Donde:

$\mathrm{L}=$ longitud de la sección del transportador $=9.53$ píe.

$\mathrm{Hp}_{\mathrm{c}}=\frac{9.53 \text { píe } * 70 \mathrm{rpm} * 18 * 4.4 * 1}{1.000 .000}$

$\mathrm{Hp}_{\mathrm{c}}=0.0528 \mathrm{hp}$.
Potencia para vencer la fricción del material en la extensión del transportador:

$\mathrm{Hp}_{\mathrm{m}}=\frac{\mathrm{C} * \mathrm{~W} * \mathrm{~L} * \mathrm{~F}_{\mathrm{m}}}{1.000 .000}$

$\mathrm{Hpm}=\underline{336 \mathrm{pie}^{3} / \mathrm{hj} * 113.51 \mathrm{Lb} / \mathrm{pí}^{3} * 9.53 \text { píe * } 2}$ 1.000 .000

$\mathrm{Hp}_{\mathrm{m}}=0.726 \mathrm{hp}$.

La potencia total es:

$\mathrm{HP}_{\mathrm{t}}=\left(\mathrm{H}_{\mathrm{p}_{\mathrm{a}}}+\mathrm{Hp}_{\underline{\mathrm{b}}}+\mathrm{Hp}_{\underline{\mathrm{c}}}+\mathrm{Hp}_{\underline{\mathrm{m}}}\right) . \mathrm{F}_{\mathrm{o}}$

Donde:

$\mathrm{F}_{\mathrm{o}}=$ factor de sobrecarga

e = eficiencia del sistema de transmisión.

De la siguiente gráfica, para $\mathrm{Hp}_{\mathrm{a}}+\mathrm{Hp}_{\mathrm{b}}+\mathrm{Hp}_{\mathrm{c}}+$ $\mathrm{Hp}_{\mathrm{m}}=1.149 \mathrm{hp}$

Se obtiene $F_{o}=1.95$

Para motorreductor con acople directo, de la tabla N4 $(\operatorname{anexo} N)$. e = 0.95. Luego:

$\mathrm{Hp}_{\mathrm{t}}=\underline{1.149 * 1.95}=2.36 \mathrm{hp}$ 0.95

Utilizando la siguiente figura, se selecciona el motor para la potencia total de $2.36 \mathrm{hp}$.

Como el alimentador de la empresa cuenta con un motor de $5 \mathrm{hp}$, no se requiere una nueva compra, sólo se recomienda realizar un mantenimiento preventivo y de limpieza y reutilizarlo para la implementación del nuevo sistema.

Cálculo y selección del reductor: En busca de la mejor eficiencia de la transmisión, mayor seguridad y menor costo de instalación y mantenimiento, y contando con el motor del que dispone la empresa, se utiliza el procedimiento de selección propuesto por industrias Ramfé Ltda ("Industrias Ramfé," 2015).

Información técnica del motor:

Marca: Siemens

Potencia: $5 / 3.7 \mathrm{hp} / \mathrm{kw}$

Corriente: $15.8-7.9$ A

Frecuencia: $60 \mathrm{~Hz}$

Velocidad: $\mathrm{n} 1=1740 \mathrm{rpm}$

Peso: $28.7 \mathrm{~kg}$

Conexionado: 220 YY - 440 YV

Tipo: Jaula de ardilla

F.P.: $\cos \Psi=0.78$

F.S.: 1.15 
En la selección del reductor se debe tener en cuenta:

En la actualidad la configuración del motorreductor del alimentador es de engranes helicoidales, por esto se continua con el mismo tipo de reductor.

Torque requerido en el eje de salida del reductor:

$\mathrm{M}_{2}=955 *\left(\mathrm{P}_{1} / \eta 2\right) * \mathrm{n}$

Donde:

$\mathrm{M}_{2}=$ Torque en el eje de salida del reductor, daN$\mathrm{m}^{1}$

$\mathrm{P}_{1}=$ Potencia a la entrada. $\mathrm{P}_{1}=3.7 \mathrm{Kw}$

$\eta_{2}=$ Velocidad requerida en el eje de salida. $\eta 2: 70$ rpm

$\eta=0.93$ (Eficiencia de la transmisión para engranes helicoidales según catálogo).

$\mathrm{M}_{2}=955 *(3.7 \mathrm{kw} / 70 \mathrm{rpm}) * 0.93=46.94 \mathrm{da} \mathrm{N}-\mathrm{m}$.

\section{Factor de servicio:}

En la tabla O1 contenida en el anexo O se obtiene:

Para carga pesada y duración del funcionamiento 8 horas/día. $\mathrm{S}_{1}=1.7$

Para carga pesada y frecuencia 2 arranques/hora $S_{2}$ $=1.0$

Luego $\mathrm{F}_{\mathrm{s}}=\mathrm{S}_{1} * \mathrm{~S}_{2}=1.7$

Factor de temperatura: para temperatura ambiente $50^{\circ} \mathrm{C}$ (considerando la irradiación de calor por la proximidad al horno) $\mathrm{F}_{\mathrm{t}}=1.6$

Como Fs es mayor a Ft, es el factor que se tiene en cuenta para la selección del reductor.

Según la tabla $\mathrm{O} 2$ contenida en el anexo $\mathrm{O}$, para la selección de reductores de engranes helicoidales se busca el valor de $\eta 2=70 \mathrm{rpm}$, se chequea un par $\mathrm{M}_{2}$ mayor al obtenido y con un fs mayor a 1.7 se obtiene el reductor de las siguientes características:

$\mathrm{P} 1=3.73 \mathrm{kw}$

$\mathrm{M} 2=47.33 \mathrm{daN}-\mathrm{m}$

Fs $=2.07$

Tamaño $=353$

Peso $=96 \mathrm{Kg}$

El factor de servicio actual $\left(\mathrm{F}_{\mathrm{s}}\right)$ es:

$$
\begin{aligned}
& \mathrm{Fs}=\underline{\mathrm{M}_{\text {admisible }}}=\underline{\mathrm{M}_{2}} * \underline{\mathrm{F}}_{\underline{\mathrm{s}}}=\underline{47.33 \mathrm{daN}-\mathrm{m} *} \\
& \underline{2.07=2.084} \underset{M_{\text {aplicación }}}{2} \mathrm{M}_{\text {aplicación }} \quad 46.94 \mathrm{daN}-\mathrm{m}
\end{aligned}
$$

\footnotetext{
${ }^{1} 1 \mathrm{da} \mathrm{N}=10 \mathrm{~N}$
}

El cual es mayor que el factor de servicio $\mathrm{Fs}=1.7$ requerido por la aplicación, por lo tanto, el reductor operará satisfactoriamente.

El pedido del reductor se efectúa con la siguiente referencia: $\mathrm{RH}-353-\mathrm{A}-4112-25-\mathrm{B} 3$

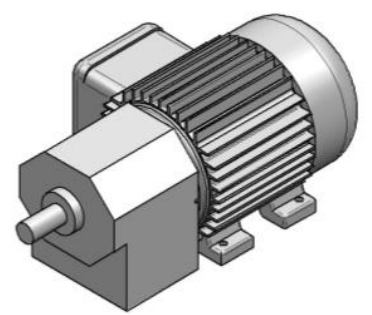

Figura 4. Conjunto motor-reductor Fuente: Elaboración propia.

\subsection{Verificación de los valores torsionales y de potencia de los componentes del alimentador}

Los tornillos alimentadores están limitados en el diseño por la cantidad de potencia y torque que pueden ser transmitidos de forma segura a través del eje hueco, acoples y pernos de sujeción.

El anexo $\mathrm{P}$ presentan las tablas con los valores de potencia y torque de los pernos, acoples y eje hueco para facilitar la comparación de los valores de todas las partes esforzadas del alimentador (Caballero A. et al., 2013).

\section{Torque transmitido:}

$\mathrm{T}=\frac{63025 * \mathrm{HP}}{\mathrm{N}}=\frac{63025 * 5 \mathrm{hp}}{70 \mathrm{rpm}}=4501.78 \mathrm{Lb}-\mathrm{pul}$ De la tabla P1 se busca el mayor valor recomendado por la norma CEMA, (7000 $>4501.78)$.

Luego se toma:

Diámetro del acople del eje $=2$ pulg

Para 2 pernos usados $=$ diámetro $=5 / 8$ pulg

Tamaño del tubo del eje $=21 / 2$ pulg

Se recomienda además usar pernos par la sujeción de cada rodamiento con un diámetro $=5 / 8$ pulg.

\section{Potencia por cada rpm:}

$\frac{5 \mathrm{hp}}{70 \mathrm{rpm}}=0.071 \mathrm{hp}$ por cada rpm

De la tabla P2 se comprueba con el mayor valor recomendado por la norma $(0.120>0.071)$ por tanto los valores tomados para los diámetros de 
acoples, pernos y tubería cumplen satisfactoriamente.

\section{Deflexión del tornillo alimentador:}

Al usar tornillos alimentadores de longitud normal, la deflexión raramente es un problema. Sin embargo, si se usan secciones más largas que las estándar, sin cojinetes intermedios, se debe tener especial cuidado para evitar que las aspas del tornillo choquen con la superficie de la artesa debido a la deflexión excesiva. La deflexión a media longitud del tornillo puede calcularse por la siguiente expresión:

$$
\mathrm{D}=\frac{5 \mathrm{WL} 3}{384(29.000 .000)(\mathrm{I})}
$$

Donde:

$\mathrm{D}=$ Deflexión en la longitud media, pulg.

$\mathrm{w}=$ Peso total del tornillo, $\mathrm{Lb}$.

$\mathrm{L}=$ Longitud del tornillo, pulg.

$\mathrm{I}=$ Momento de inercia del eje

Según el catálogo, para un diámetro de 6 pulg y una longitud de 10 pies, el peso máximo del tornillo es $\mathrm{w}=150 \mathrm{lb}$.

$\mathrm{L}=13.53$ píe $=162.36$ pulg.

Según la tabla 16 para material Sch 40 (recomendado) y el diámetro del eje de 2 1/2 pulg., el momento de inercia $\mathrm{I}=1.53$

Reemplazando los valores se obtiene:

$$
\mathrm{D}=\frac{5 * 210 \mathrm{Lb} *(162.36 \mathrm{pulg})^{3}}{384(29.000 .000)(1.53)}=0.188 \text { pulg. }
$$

La deflexión obtenida es menor a 0.25 pulg. (1/4"), la cual es la deflexión crítica, por esto se deduce que el alimentador no sufre problemas de atascos ni obstrucciones entre las aspas y la artesa.

\subsection{Selección de los componentes estándar del sistema}

A continuación se selecciona los principales componentes de acuerdo a la serie de selección: grupo 3, mediante el uso de la tabla Q1 (anexo Q) se obtiene:

Para diámetro de 6 pulg:

Número de tornillo: para aspa seccional: 6\# 312, debido a que ésta es la especificación para la sección de extensión del transportador
Espesor de la artesa: para mayor seguridad se toma $3 / 16$ pulg.

Con el número del tornillo, se selecciona el resto de los componentes:

Tipo de encerramiento (tapa) de la artesa: existen dos clases:

Clase 1: son proporcionados principalmente para la protección del personal que opera el equipo, o donde el encerramiento forma parte funcional del alimentador o la estructura.

Clase 2: se construye para proveer alguna medida de protección contra el polvo que pueda levantarse o para proteger el mismo material.

Debido a que el material es húmedo, que no existen agentes externos que puedan contaminarlo y a la implementación de una buena señalización de riesgo para evitar accidentes (ver Manual de operación, mantenimiento y seguridad), se decide no colocar una tapa fija a la artesa.

Ubicación de los pernos en la pestañas de los extremos de la artesa.

Para artesa en forma de U y diámetro del alimentador de 6 pul se recomienda usar 6 pernos, con un diámetro de $3 / 8$ pulg.

Ancho $=\mathrm{A}=7$ pulg

Altura media $=\mathrm{B}=3 \frac{5}{8}$ pulg. Además se presentan las distancias de espaciamiento recomendada entre pernos.

Especificaciones para los pernos de sujeción: A continuación se presentan las dimensiones requeridas para los pernos que sujetan los accesorios que componente la estructura del alimentador.

Pestañas de la artesa: 6 pernos, de $3 / 8 \times 1$ "

Tamaño, dimensiones $\mathbf{y}$ peso del eje: de la tabla Q2 (anexo Q) se extrae las dimensiones recomendadas para el diámetro del eje de $21 / 2$ pulg.

Diámetro exterior: 2.875 pulg.

Material recomendado: Sch 40

Espesor de pared: $\mathrm{e}=0.203$ pulg.

Diámetro interior: 2.469 pulg

Peso del eje: $5.793 \mathrm{Lb} /$ pie; como $\mathrm{L}=13.53$ pie

Weje $=78.37 \mathrm{Lb}$

Especificaciones para las aspas del alimentador: de la tabla Q3 del anexo Q según recomendación de la norma CEMA, para diámetro del alimentador de 6 pulg.

Diámetro del acople: $1 \frac{112}{2}$ pulg. 
Para tornillo 6 \#312 y material Sch 40, los espesores del aspa son:

Espesor interior: 3/8 pulg.

Espesor exterior: $3 / 16$ pulg.

\section{Especificaciones de la artesa:}

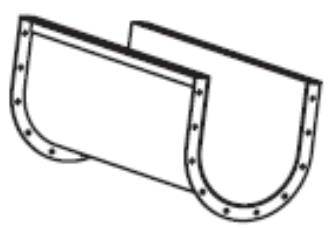

Figura 5.Tipo de artesa utilizada.

Fuente: Elaboración propia.

Comúnmente se utiliza una artesa tipo económico en forma de U (lámina doblada). Las especificaciones de las dimensiones de construcción son:

Material: lámina de acero resistencia de la abrasión.

Espesor de la lámina $=\mathrm{e}=31 / 6$ pulg.

Ancho de la artesa $=\mathrm{A}=7$ pulg.

Radio de la curvatura $=\mathrm{A} / 2=3.5$ pulg.

Ancho de la pestaña (igual pestaña para la percha) $=\mathrm{B}=1 \frac{1}{2}$ pulg.

Distancia del centro del eje al borde superior de la artesa $=\mathrm{C}=4 \mathrm{1} / 2$ pulg.

Longitud total de la artesa $=13.53$ píe

\section{Especificaciones del pico de descarga:}

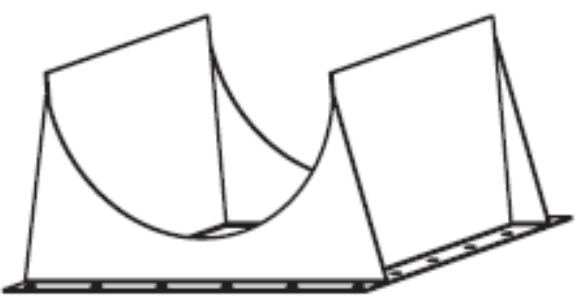

Figura 6. Pico de descarga utilizado. Fuente: Elaboración propia.

La configuración anterior es la más utilizada y recomendada por la norma CEMA. El espesor se toma igual al de la artesa. e = 316 pulg. Para diámetro del alimentador de 6 pulg., se recomiendan las siguientes dimensiones:

Ancho del pico (igual ancho de la artesa) $=\mathrm{A}=7$ pulg.

Distancia del centro del pico al extremo de la artesa $=\mathrm{B}=6$ pulg.

Altura del pico $=\mathrm{C}=5$ pulg.

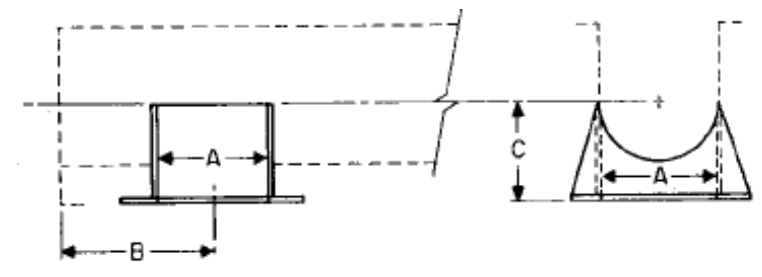

Figura 7. Dimensiones del tipo de descarga.

Fuente: Elaboración propia.

\section{Especificaciones de las Perchas o extremos de la} artesa:

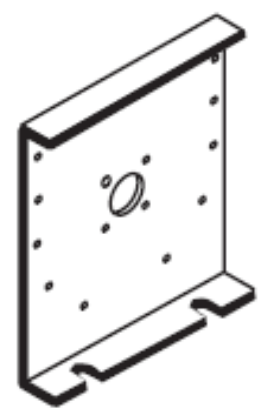

Figura 8. Tipo de percha utilizada. Fuente: Elaboración propia.

Es el tipo más común y el más recomendado como percha, el rodamiento se sujeta a la pecha por medio de una brida atornillada (Rodríguez $\mathrm{O}$ et al., 2012).

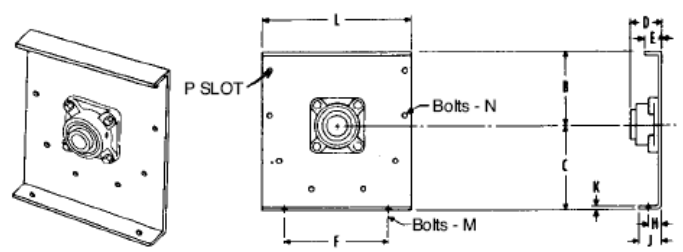

Figura 9. Dimensiones típicas para las perchas.

Fuente: Elaboración propia.

Para diámetro del alimentador de 6 pulg., las dimensiones recomendadas son:

Diámetro del acople eje tubo: $1 \frac{1}{2}$ pulg.

Ancho de la percha: $\mathrm{L}=10$ 1/8 pulg.

Distancia del centro del eje al borde superior $=\mathrm{B}=$ $4 \frac{1}{2}$ pulg.

Tolerancia entre el extremo del eje y la percha, D para rodamiento de rodillos, $\mathrm{D}=311 / 16 \mathrm{pulg}$.

Distancia entre los pernos del píe de la percha $=\mathrm{F}$ $=81 / 8$ pulg.

Distancia placa de sujeción del rodamiento a la percha $=\mathrm{H}=1$ pulg.

Pestaña superior de 1 percha $=E=1 \frac{1}{2}$ pulg.

Distancia del centro del eje al borde interior $=\mathrm{C}=$ 5 5/8 pulg.

Espesor de la lámina de la percha $=\mathrm{K}=1 / 4$ pulg. 
Pestaña inferior de la percha $=\mathrm{J}=13 / 4$ pulg.

Diámetro de los pernos de sujeción a la artesa $=\mathrm{N}$ $=3 / 8$ pulg.

Diámetro de los pernos de anclaje de la percha $=\mathrm{M}$ $=3 / 8$ pulg.

Dimensión de los agujeros $=\mathrm{P}=7 / 16 * 9 / 16$

Soporte de la pecha y la artesa en los extremos:

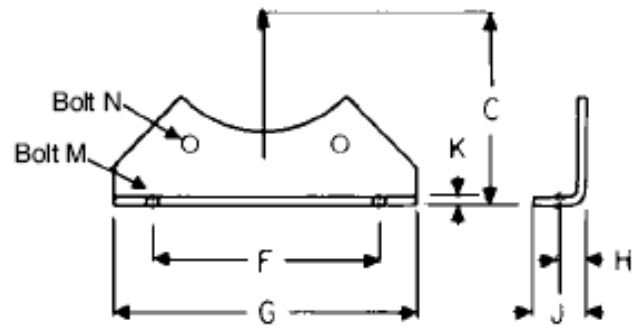

Figura 10. Dimensiones de los soportes de las perchas. Fuente: Elaboración propia.

Las dimensiones recomendadas son:

$\mathrm{C}=55 / 8$ pulg

$\mathrm{F}=81 / 8$ pulg

$\mathrm{G}=10$ pulg

$\mathrm{H}=1 \frac{1}{4}$ pulg

$\mathrm{K}=1 / 4$ pulg

$\mathrm{K}=3 / 16$ pulg.

Para los soportes intermedios de la artesa se utilizan soportes de píe en madera para mayor economía, dándoles la forma de U de la artesa.

Selección de los rodamientos de soporte del eje: para el análisis de las fuerzas que determinan las cargas que actúan sobre los rodamientos, se analiza el eje del alimentador.

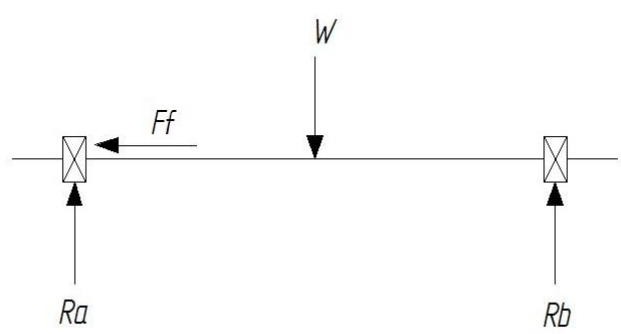

Figura 11. Cargas que actúan sobre el eje del alimentador.

Fuente: Elaboración propia.

$\mathrm{W}=$ Peso total del tornillo $=$ peso eje + peso aspas

$\mathrm{F}_{\mathrm{f}}=$ Fricción de arrastre del material $=$ masa $* \mathrm{~g} *$

$\mu_{\mathrm{f}}$

$\mathrm{F}_{\mathrm{f}}=\mathrm{N} . \mu_{\mathrm{f}}$

$\mathrm{N}=$ m.g.

Analizando como tornillo de potencia:

$\mathrm{m}=(\text { capacidad } / \text { revolución })^{*} \rho$ material $* \mathrm{n}$ pasos

Donde:
$\mathrm{C} / \mathrm{rev}=\frac{\mathrm{C}}{\operatorname{Rev} / \text { hora }}$

$\mathrm{C} / \mathrm{rev}=\frac{348 \mathrm{píe} 3 / \text { hora }}{70 \mathrm{rev} / \mathrm{min}=4200 \mathrm{rev} / \mathrm{h}}=0.082 \mathrm{pí}^{3} / \mathrm{rev}$

$\mathrm{C} / \mathrm{rev}=2.32 \times 10^{-3} \mathrm{~m}^{3} / \mathrm{rev}=2323.76 \mathrm{~cm}^{3} / \mathrm{rev}$

Ahora: se determina el número de pasos de todo el tornillo

Sección $1 / 2$ paso: $\mathrm{n}$ pasos $=(36 \mathrm{Pul} / 3 \mathrm{Pul})=12$ pasos

Sección $2 / 3$ paso: $\mathrm{n}$ pasos $=(12 \mathrm{Pul} / 4 \mathrm{Pul})=3$ pasos

Sección Paso estándar: $\mathrm{n}$ pasos $=(114,24$ Pul -6 Pul) $=18.04$ pasos

Numero de pasos es igual a 33, de lo que resulta:

$\mathrm{m}=2.32 \times 10^{-3} \mathrm{~m}^{3} * 1830 \mathrm{~kg} / \mathrm{m}^{3} * 33$ pasos

$\mathrm{mg}=140.10 \mathrm{~kg} * 9.8 \mathrm{~m} / \mathrm{s}^{2}=1374.38 \mathrm{~N}$

Como $\mathrm{N}=\mathrm{mg}$

$\mathrm{F}_{\mathrm{f}}=\mathrm{N} * \mu$

Con $\mu=0.4125$ (arena contra el metal)

$\mathrm{F}_{\mathrm{f}}=0.4125 * 1374.38 \mathrm{~N}$

$\mathrm{F}_{\mathrm{f}}=566.93 \mathrm{~N}$

$\mathrm{m}_{\text {tornillo }}=150 \mathrm{lb}=68.03 \mathrm{~kg}$

$\mathrm{w}_{\text {tornillo }}=667.37 \mathrm{~N}$

Del d.c.l. del eje se observa que:

$\mathrm{R}_{\mathrm{A}}=\mathrm{R}_{\mathrm{B}}=\mathrm{w} / 2=283.46 \mathrm{~N}$

Por medio del catálogo de selección de SKF, trabajando en fase a la confiabilidad del $90 \%$, se tiene:

Para máquinas de 8 horas de trabajo no utilizadas totalmente:

Horas de servicio $=\mathrm{L}_{10} \mathrm{~h}=10.000-25.000$, tomando $\mathrm{L} 10 \mathrm{~h}=15.000$

Duración nominal $=\mathrm{L}_{10}=15.000$ horas $* 60$ $\mathrm{min} / 1$ hora $* 70 \mathrm{rev} / 1 \mathrm{~min}=63$ millones $\mathrm{de}$ revoluciones

Selección del rodamiento en A:

Carga axial $=566.93 \mathrm{~N}$

Carga radial $=283.46 \mathrm{~N}$

$\mathrm{Fa}=566.93 \mathrm{~N}=2$

Fr $283.46 \mathrm{~N}$ 
$\varnothing$ eje $=2 \frac{1}{2} "=63.5 \mathrm{~mm}$

Se toma rodamiento de cilindros abombados:

Para $\mathrm{d}=2 \frac{1}{2}$ “

$\mathrm{D}=130 \mathrm{~mm}$

$\mathrm{B}=31 \mathrm{~mm}$

$\mathrm{C}=30500 \mathrm{Lb}=135.670 \mathrm{~N} \mathrm{Co}=24.500 \mathrm{Lb}=$

$108981,15 \mathrm{~N}$

$\underline{\mathrm{Fa}}=\underline{567 \mathrm{~N}}=0.0052$

Fr $108981 \mathrm{~N}$

$\mathrm{e}=0.22$

$\mathrm{Y}$ para $\mathrm{Fa} / \mathrm{Fr}>\mathrm{e}$

$\mathrm{x}=0.67$

$\mathrm{y}=4.6$

Se calcula la carga dinámica equivalente:

$\mathrm{P}=\mathrm{XF}_{\mathrm{r}}+\mathrm{YF}_{\mathrm{a}}$

$\mathrm{P}=0.67 * 283.46 \mathrm{~N}+4.6 * 566.93 \mathrm{~N}$

$\mathrm{P}=2797.79 \mathrm{~N}$

$\mathrm{L}_{10}=$ Duración nominal (millones de revoluciones)

$\mathrm{n}=$ factor de rodamiento

$\mathrm{n}=10 / 3 \rightarrow$ rod. de rodillos

Luego:

$\mathrm{C} *=(63)^{1 / 10 /}{ }_{3} * 2797.79 \mathrm{~N}$

$\mathrm{C}=9696.55 \mathrm{~N}=43132.23 \mathrm{Lb}$

Como $\mathrm{C}>\mathrm{C} *$, rodamiento en $\mathrm{B}$ se selecciona con las mismas características:

Designación:

Selección del soporte del rodamiento: Según recomendación del catálogo Martín, el soporte es de tipo brida atornillado, seleccionado por el catálogo de SKF (SKF, 2015), se tiene para tipo Bd1 > $60 \mathrm{~mm}$.

Para $\mathrm{d} 1=65 \mathrm{~mm}$

Designación $=722515 \mathrm{~B}$

Para C $=30.500 \mathrm{Lb}$, se selecciona el soporte con su adaptador, cuya designación es $=22215 \mathrm{~K}+\mathrm{H} 315$.

\section{CONCLUSIONES}

La implementación del sistema alimentador en la planta de producción permite mejorar el flujo de materiales producidos hacia el horno de secado, aumentando la productividad y reduciendo costos de operación.

El acondicionamiento del terreno y específicamente la zona de carga, mantienen la calidad del producto y evita desperdicio de material.

Con la construcción del muro y la base en concreto reforzado se obtiene mayor estabilidad en los equipos, lo que se refleja en la alineación, el funcionamiento silencioso y en una mayor vida útil de los mismos.

\section{REFERENCIAS}

Budynas, R. G., \& Nisbett, J. K. (2008). Diseño en Ingeniería Mecánica. (MacGrawHil, Ed.) (MacGrawHil). Mexico.

Caballero Amaury, Velasco Gabriel, Pardo García A. (2013). DIFFERENTIATIONS OF OBJECTS IN DIFFUSE DATABASES. Revista colombiana de tecnologías de Avanzada. 2 (22). Pág. 131 - 137

García-León, R. A., Flórez Solano, E., \& Acosta Pérez, M. A. (2015). Análisis estructural de una máquina prensadora para producción de ladrillo macizo para las pequeñas industrias artesanales de materiales cerámicos en Ocaña Norte de Santander y en la región. Revista Colombiana de Tecnologías de Avanzada, 1(1692-7257), 7.

Industrias Ramfé. (2015). Retrieved from http://www.ramfe.com.co/

Jaeger, H. M., Nagel, S. R., Behringer, R. P., Hugo, V., \& Miserables, L. (1996). The physics of granular materials. Physics Today, 49(April), 31-38.

Knowlton, T. M., Klinzing, G. E., \& Yang, W. C. (1994). The importance of storage, transfer, and collection. Chemical Engineering Progress, 1, 1-10. Retrieved from https://www.osti.gov/scitech/biblio/7036222

Link-Belt Cranes. (2015). Retrieved from https://www.link-belt.com/

Marinelli, J., \& Carson, J. W. (1992). Solve solids flow problems in bins, hoppers, and feeders. Chemical Engineering Progress.

Martin. (2015). Retrieved from http://www.martinsprocket.com/

Nedderman, R. M. (2005). Statics and Kinematics of Granular Materials. Estados Unidos: Cambridge University Press. Retrieved from https://books.google.com.co/books?id=52Xu yGlewh8C.

Rodríguez Oscar Oswaldo, Pineda Pinto Ronald Fernando, Cárdenas Pedro Fabián. (2012). Herramientas EJS 3D/MATLAB para el control del sistema no lineal aplicado al péndulo invertido sobre carro deslizante. Revista colombiana de tecnologías de Avanzada. 1 (19). Pág. 28 - 34.

SKF. (2015). Rodamientos. Estados Unidos.

Torres Clayton José, Archila John Faber, Tronco Mário Luiz, Becker Marcelo, Viera Porto Arthur José, Tiberti Alexander José. (2013). Estudio cinemático de una plataforma robótica para agricultura. Revista colombiana de tecnologías de Avanzada. 2 (22). Pág. 131 $-137$. 